\title{
Actividad sexual en adolescencia temprana: problema de salud pública en una ciudad colombiana
}

\author{
Luís Alfonso Mendoza T. 1,3, Martha Arias G. ${ }^{2}$, Marly Pedroza P. ${ }^{3}$, Paul Micolta $C^{3}{ }^{3}$, \\ Andrés Ramírez $R^{3}$, Christian Cáceres G. ${ }^{3}$, Darling López $S^{3}{ }^{3}$, Antonio Núnez G. ${ }^{3}$, \\ Maria Acuña $P .^{3}$ \\ ${ }^{1}$ División de Epidemiología Hospitalaria, ${ }^{2}$ Departamento de Pediatría; Fundación Hospital San José de Buga, Colombia. \\ 3 Unidad Central del Valle, Tuluá, Colombia.
}

\section{RESUMEN}

Antecedentes: El inicio de la actividad sexual en adolescentes, está asociada a pobre planificación familiar (PF), mayor tasa de fecundidad y riesgo de adquisición de enfermedades de transmisión sexual (ETS). Objetivo: Evaluar el impacto sobre la fecundidad, planificación familiar y lesiones de cuello uterino en una población de adolescentes y jóvenes, que iniciaron actividad sexual en la adolescencia. Método: Estudio de cohorte retrospectiva. Incluyó 845 adolescentes y jóvenes que iniciaron actividad sexual en la adolescencia y que consultaron a una Institución Prestadora de Servicios de Salud en Tuluá, Colombia. El análisis incluyó estadísticas descriptivas, análisis de riesgo relativo (RR) y atribuible a la exposición (RAexp) expresado en porcentaje, como indicadores de asociación. Resultados: Hubo 203 adolescentes y 642 jóvenes. El promedio de edad de inicio de la actividad sexual fue 16 años ( $\pm 1,6$ años). El $49 \%$ no realizaba $P F, 34,7 \%$ tenía $\geq 1$ hijo y $6,9 \%$ alguna lesión en cuello uterino. Hubo asociación entre actividad sexual en la adolescencia temprana y tener un hijo, al compararlo con el inicio de la actividad sexual en la adolescencia media (RR: 1,6; IC 95\%: 1,2-2,1. RAexp\%: 35,7\%; IC 95\%: 13,3-52,4\%) y tardía (RR: 2; IC 95\%: 1,5-2,6. RAexp\%: 49\%; IC 95\%: 31,7-62\%). Conclusiones: Nuestros resultados confirman el inicio precoz de la actividad sexual en adolescentes, el bajo uso de anticonceptivos y el riesgo de lesiones cervicales. Programas educativos conducentes al retraso del inicio de la actividad sexual, el uso de métodos anticonceptivos efectivos y de barrera, permitirán reducir las cifras encontradas en este estudio.

\section{PALABRAS CLAVE: Adolescencia, planificación familiar}

\section{SUMMARY}

Background: The onset of sexual activity in adolescents is associated with poor family planning, a higher fertility rate and risk of acquiring sexually transmitted diseases. Objective: To evaluate the impact on fertility, family planning and cervical lesions in a population who initiated sexual activity in adolescence. Method: A retrospective cohort study. Included 845 adolescents and young people who initiated sexual activity during adolescence and who consulted a Lender Institution Health Services in Tuluá, Colombia. The analysis included descriptive statistics, analysis of relative risk (RR) and attributable to exposure (RAexp) expressed in percentage, as indicators of association. Results: There were 203 adolescents and 642 young people. The average age of onset of sexual activity was 16 ( \pm 1.6 years). $49 \%$ did not perform family planning, $34.7 \%$ had $\geq 1$ child and $6.9 \%$ cervical lesions. There was an association between sexual activity in early adolescence and have a child, when compared with the onset of sexual activity in middle (RR: $1.6,95 \% \mathrm{Cl} 1.2$ to 
2.1. RAexp\%: $35.7 \%, 95 \% \mathrm{Cl}: 13.3$ to $52.4 \%$ ) and late adolescence (RR: $2,95 \% \mathrm{Cl}: 1.5$ to 2.6 . RAexp\%: $49 \%, 95 \% \mathrm{Cl}: 31.7-62 \%)$. Conclusions: Our results confirm the early onset of sexual activity in adolescents, low contraceptive use and risk of cervical lesions. Educational programs leading to delayed onset of sexual activity, the use of effective contraceptive methods and barrier methods will reduce the numbers found in this study.

\section{KEY WORDS: Adolescence, family planning}

\section{INTRODUCCIÓN}

Adolescencia según la OMS es el período de la vida en el cual el individuo adquiere la capacidad reproductiva, transita los patrones psicológicos de la niñez a la adultez y consolida la independencia socioeconómica. Sus límites han sido fijados entre los 10 y 19 años (1-6).

A la adolescencia se la puede dividir en tres etapas, con características diferentes y a su vez con formas distintas de enfrentar la sexualidad y un embarazo: 1. Adolescencia temprana (10 a 14 años); 2. Adolescencia media (15 a 16 años) y 3 . Adolescencia tardía (17 a 19 años) $(1,7)$.

La primera relación sexual cada vez ocurre en estadios de la vida más tempranos y mientras más precoz ocurre el primer coito, mayor es el número de parejas sexuales que tienen esas personas y por lo tanto los riesgos se multiplican (embarazos, ETS) (8). Las estadísticas nos muestran que el inicio de la actividad sexual (AS) ocurre alrededor de los 16 años (9), con un creciente índice de embarazos en adolescentes y la presencia de SIDA en personas jóvenes. El embarazo no planeado en esta etapa de la vida, se asocia con el inicio temprano de relaciones sexuales y el uso inconsistente de métodos anticonceptivos, incluyendo el condón (10).

En cuanto al uso de anticoncepción en la Región, se ha observado una tendencia constante al aumento del uso de estos. Langer (11), describe que en países como Bolivia (uno de los países con una prevalencia de uso más baja) la proporción de mujeres en edad fértil que utiliza anticoncepción ha aumentado del $30,3 \%$ en 1989 al $45,3 \%$ en 1994 y al $48,3 \%$ en 1998; y en Nicaragua del $49 \%$ en 1993 al $60,3 \%$ en 1998 . En el otro extremo del espectro (países con frecuencias de uso elevadas), Colombia muestra un aumento del $72 \%$ en 1995 al $77 \%$ en 2000 , y en México se observa una tendencia ascendente sin interrupciones: del $63,1 \%$ en 1992 al $66,5 \%$ en 1996 y al $70,8 \%$ en 2000 (11).

El impacto del embarazo en la adolescencia es psicosocial y se traduce en deserción escolar, mayor número de hijos, desempleo, fracaso en la relación de pareja e ingresos inferiores de por vida. Contribuye a perpetuar el ciclo de la pobreza y la "feminización de la miseria" (7). El embarazo en la adolescencia representa un serio problema social (12), y se considera desde el punto de vista médico y social como una situación de riesgo (13). Países como Chile reportaron en 2005, 230.831 nacidos vivos, $935(0,41 \%)$ correspondieron a embarazos de adolescentes de 10-14 años y 35.143 $(15,2 \%)$ a adolescentes de 15-19 años, 5 de estas últimas fallecieron como consecuencia del proceso reproductivo $(14,2 / 100.000 \mathrm{nv})$. La mortalidad materna en adolescentes menores, pese a su elevada tasa $(41,9 / 100.000 \mathrm{nv})$ no fue significativa y en adolescentes mayores (19,3/100.000 nv) fue significativamente menor al grupo control, explicado especialmente por una baja mortalidad por aborto $(3,9 / 100.000$ nv) (14). Para Chile, al comparar el año 2004 (año con la natalidad más baja desde 1990), con los años 2005 y 2006, observaron un discreto aumento de la natalidad general acumulada de $0,45 \%$. El problema radicó, en que esta natalidad aumentó en las adolescentes $(5,3 \%$ para las menores de 15 años y 9,6\% para las de $15-19$ años) y en mujeres en edad reproductiva avanzada (28,5\% para las mujeres de 45 o más años) (15).

El objetivo de este estudio es evaluar el impacto y asociación sobre la fecundidad, planificación familiar y presencia de lesiones de cuello cervicouterino que tiene el inicio de la AS en la adolescencia, en una población de adolescentes y jóvenes que consultaron a una Institución Prestadora de Servicios de Salud (IPS) para tamización de cáncer de cuello uterino, entre 2008 y 2011 en Tuluá, Colombia.

\section{PACIENTES Y MÉTODOS}

Tipo de estudio. Estudio de cohorte retrospectiva realizado en mujeres que consultaron a una IPS (UniCáncer, Capítulo Tuluá, Colombia) para toma de citologías vaginales, entre los años 2008 y 2011. No hubo intervenciones y la información fue tomada de una base de datos que se lleva en dicha institución. 
Muestreo y tamaño de muestra. La muestra está constituida por todas las adolescentes y jóvenes que iniciaron la $A S$ en la adolescencia temprana, media y tardía ( $\mathrm{n}: 845)$. El muestreo fue no probabilístico, incluyendo al estudio todas las personas que cumplieron con los criterios de inclusión. Con este tamaño de muestra se tuvo para un Riesgo Relativo de 1,4 , un nivel de confianza del $95 \%$ y un poder del $80 \%$. Se incluyeron adolescentes y jóvenes entre los 14 y 25 años que asistieron a tamización para cáncer de cuello mediante citología de cuello uterino, residentes en el municipio de Tuluá, Colombia y que habían iniciado la AS en la adolescencia (10-19 años). Se excluyeron las personas con ausencia de variables en los registros, en estado de gestación, con infecciones vaginales, histerectomizadas y personas con conizaciones antes del 2008 que pudieran alterar los resultados citológicos.

Exposiciones. Se consideró exposiciones fijas la edad de inicio de la AS. Para ello se establecieron tres grupos: adolescencia temprana (10-14 años), media (15-16 años) y tardía (17-19 años).

Evento a determinar. Los eventos a determinar fueron número de partos, métodos de planificación familiar (PF) y lesiones de bajo y alto grado de cuello de útero, determinado mediante la citología de cuello cervicouterino empleando la clasificación "Bethesda System" (16).

Fuente de información. La información fue obtenida de los registros de citologías realizados entre 2008 y 2011 por UniCáncer, Capítulo Tuluá, Colombia, lugar donde acuden mujeres para la toma de citología de cuello de útero. El método de recolección que se utilizó fue la extracción directa de los datos de dichos registros, previa capacitación y entrenamiento del personal.

Análisis estadístico. Para el análisis estadístico se utilizó el paquete estadístico Stata $\otimes_{11.0}$. Se obtuvieron estadísticas descriptivas de las variables de interés, empleando medidas de tendencia central y de dispersión (promedios con su desviación estándar o medianas con sus cuartiles 1 y 3) para las variables continuas según su distribución, siendo comparadas mediante la prueba t-test no pareado o Wilcoxon rank-sum (Prueba de Mann-Whitney). Las variables agrupadas en categorías se expresaron en frecuencias y proporciones y se compararon mediante la prueba Chi2 de Pearson con un nivel de significancia de $\mathrm{p}<0,05$.

Análisis estadístico bivariado. La independencia entre las variables se evaluó mediante el test de Chi2 de Pearson. Las asociaciones se estimaron mediante el RR y el RAexp expresada en porcentaje, con sus intervalos de confianza de $95 \%$ (IC $95 \%)$. Para el grado de asociación entre las variable categórica se utilizaron pruebas de Chi2, prueba de Chi2 con corrección de Yates y prueba exacta de Fisher con un nivel de significancia de $p<0,05$.

Aspectos éticos. Para la realización de esta investigación se contó con la autorización del Comité de Ética de la Institución, y según la legislación colombiana ésta se trata de una investigación "sin riesgo". La investigación carece de conflicto de intereses desde el punto de vista de patrocinios o remuneración alguna.

\section{RESULTADOS}

Se incluyeron un total de 845 mujeres, 203 (24\%) adolescentes y 642 (76\%) jóvenes, que habían iniciado la AS en la adolescencia (Tabla I). El promedio de la edad de inicio de la AS fue de 16 años $( \pm 1,6$ años). Entre las que iniciaron la AS en la adolescencia temprana ( $<15$ años), esta ocurrió a los 13,5 años $( \pm 0,7$ años), mientras que aquellos que la iniciaron en la adolescencia media (15-16 años) lo hicieron a los 15,5 años ( $\pm 0,5$ años) y en la adolescencia tardía (17-19 años) a los 17,7 años $( \pm 0,7)$ (Tabla I).

El $49 \%$ de las adolescentes y jóvenes no planificaban, mientras los métodos más usados en el $51 \%$ restante fueron: hormonal $34 \%$, de barrera $5 \%$, ligadura de trompas $4,6 \%$, otros métodos $4,7 \%$, dispositivo intrauterino (DIU) 2,6\% y vasectomía $0,1 \%$. Hubo un mayor uso de los métodos hormonales en los que iniciaron la AS en la adolescencia media y tardía $(p<0,05)$ (Tabla I).

El $65,3 \%$ de las participantes del estudio no habían tenido hijos al momento de la consulta, mientras el $25,9 \%, 7 \%$ y $1,8 \%$ habían tenido 1,2 y $\geq 3$ hijos respectivamente. El $48,4 \%$, $66 \%$ y $70,9 \%$ de los que iniciaron la AS en la adolescencia temprana, media y tardía respectivamente no habían tenido hijos, diferencia estadísticamente significativa $(p<0,05)$. El $15,9 \%, 6,9 \%$ y $3,8 \%$ de las que iniciaron la AS en la adolescencia temprana, media y tardía respectivamente, habían tenido 2 hijos, diferencia estadísticamente significativa $(p<0,05)$, mientras que la multiparidad ( $\geq 3$ hijos) se presentó con más frecuencia en aquellas mujeres que habían iniciado la AS en la adolescencia temprana, comparado con las media y la tardía $(6,3 \%, 1,6 \%$ y $0,3 \%$ respectivamente; $p<0,05$ ) (Tabla I).

En el $4,9 \%$ de los adolecentes y jóvenes se presentaron lesiones de bajo grado, mientras las lesiones de alto grado correspondieron al $0,7 \%$ y otras lesiones $1,3 \%$. No se encontró neoplasia maligna del cuello cervicouterino. Sin embargo, no se halló diferencia estadísticamente significativa para los diferentes tipos de lesiones en los tres grupos evaluados (Tabla I). 
Tabla I

DATOS DEMOGRÁFICOS, SEXARQUIA, PARIDAD, PLANIFICACIÓN FAMILIAR Y LESIONES EN CUELLO UTERINO

\begin{tabular}{|c|c|c|c|c|c|}
\hline Características & $\begin{array}{c}\text { Todas las } \\
\text { personas } \\
\mathrm{n}: 845\end{array}$ & $\begin{array}{c}\text { Sexarquia en } \\
\text { adolescencia } \\
\text { temprana } \\
\text { n: } 126\end{array}$ & $\begin{array}{c}\text { Sexarquia en } \\
\text { adolescencia } \\
\text { media } \\
\text { n: } 379\end{array}$ & $\begin{array}{c}\text { Sexarquia en } \\
\text { adolescencia } \\
\text { tardía } \\
\text { n: } 340\end{array}$ & Valor $p$ \\
\hline $\begin{array}{l}\text { Edad (años) } \\
\text { Promedio (DE) }\end{array}$ & $21,6( \pm 2,5)$ & $21( \pm 3)$ & $21( \pm 2,4)$ & $22,3( \pm 2,2)$ & $>0,05$ \\
\hline $\begin{array}{l}\text { Sexarquia (años) } \\
\text { Promedio (DE) }\end{array}$ & $16,1( \pm 1,6)$ & $13,5( \pm 0,7)$ & $15,5( \pm 0,5)$ & $17,7( \pm 0,7)$ & $<0,05$ \\
\hline $\begin{array}{l}\mathrm{N}^{\circ} \text { de partos } \\
\text { Promedio (DE) }\end{array}$ & $0(0-1)$ & $1( \pm 1)$ & $0,5( \pm 0,8)$ & $0,3( \pm 0,6)$ & $<0,05$ \\
\hline Planificación familiar : & n (\%) & n (\%) & n (\%) & n (\%) & \\
\hline Ninguno & $414(49)$ & $59(46,8)$ & $185(48,8)$ & $170(50)$ & \\
\hline DIU & $22(2,6)$ & $7(5,6)$ & $8(2,1)$ & 7 (2) & \\
\hline Vasectomia & $1(0,1)$ & $0(0)$ & $1(0,3)$ & $0(0)$ & \\
\hline Ligadura & $39(4,6)$ & $13(10,3)$ & $20(5,3)$ & $6(1,8)$ & \\
\hline Hormonal & $287(34)$ & $34(27)$ & $130(34,3)$ & $123(36,2)$ & \\
\hline Barrera & $42(5)$ & $5(4)$ & $17(4,5)$ & $20(5,9)$ & \\
\hline Otro & $40(4,7)$ & $8(6,4)$ & $18(4,7)$ & $14(4,1)$ & $>0,05$ \\
\hline $\mathrm{N}^{\circ}$ de partos: & $\mathrm{n}(\%)$ & $\mathrm{n}(\%)$ & n (\%) & n (\%) & \\
\hline 0 & $552(65,3)$ & $61(48,4)$ & $250(66)$ & $241(70,9)$ & \\
\hline 1 & $219(25,9)$ & $37(29,4)$ & $97(25,6)$ & $85(25)$ & \\
\hline 2 & $59(7)$ & $20(15,9)$ & $26(6,9)$ & $13(3,8)$ & \\
\hline$\geq 3$ & $15(1,8)$ & $8(6,3)$ & $6(1,6)$ & $1(0,3)$ & $<0,05$ \\
\hline Lesiones de cuello uterino: & n (\%) & n (\%) & $\mathrm{n}(\%)$ & n (\%) & \\
\hline Ninguno & $787(93,1)$ & $119(94,4)$ & $354(93,4)$ & $314(92,4)$ & \\
\hline Bajo grado & $41(4,9)$ & $5(4)$ & $17(4,5)$ & $19(5,6)$ & \\
\hline Alto grado & $6(0,7)$ & $1(0,8)$ & $2(0,5)$ & $3(0,9)$ & \\
\hline Otras & $11(1,3)$ & $1(0,8)$ & $6(1,6)$ & $4(1,2)$ & $>0,05$ \\
\hline
\end{tabular}

Lesiones de bajo grado: NIC I. Lesiones de alto grado: NIC II, NIC III, cáncer in situ, neoplasia maligna de cuello uterino. Otras: Atipia en células escamosas de significado indeterminado (ASC-US ), Atipia en células escamosas significado indeterminado sugestivo (ASCUS-H), Anormalidades de células glandulares (ASGUS)

El inicio de la AS en la adolescencia temprana aumenta el riesgo de embarazos en la propia adolescencia y periodo juvenil, al compararlas con las que inician la AS en la adolescencia media (RR: 1,6; IC 95\%: 1,2-2,1. RAexp\%: 35,7\%; IC 95\%: $13,3-52,4 \%$ ) y adolescencia tardía (RR: 2; IC 95\%: 1,5-2,6. RAexp\%: 49\%; IC 95\%: 31,7-62\%) (Tablas II y III). Mientras el riesgo de tener más de un em- barazo se incrementa entre más tempranamente se inicien las relaciones sexuales, y la multiparidad ( $\geq 3$ hijos) es más frecuente en aquellas niñas que inician la AS por debajo de los 15 años, al compararlas con las niñas de 15-16 años (RR: 2,9; IC 95\%: 1,8-4,8. RAexp\%: 65,7\%; IC 95\%: 43-79,3\%) y 17-19 años (RR: 4,4; IC 95\%: 3,2-6,1. RAexp\%: 77,3\%; IC 95\%: 68,6-83,5\%) (Tablas II y III). 
Tabla II

EMBARAZOS, PARTOS Y LESIONES DE CUELLO UTERINO EN ADOLESCENCIA TEMPRANA VS MEDIA

\begin{tabular}{|c|c|c|c|c|c|}
\hline Resultados & $\begin{array}{c}\text { Sexarquia en } \\
\text { adolescencia } \\
\text { temprana } \\
\text { n: } 126\end{array}$ & $\begin{array}{c}\text { Sexarquia en } \\
\text { adolescencia } \\
\text { media } \\
\text { n: } 379\end{array}$ & $\mathrm{RR}$ & RAexp \% & $\begin{array}{c}\text { Valor } \\
\mathrm{p}\end{array}$ \\
\hline Embarazo n (\%) & $65(51,6 \%)$ & $129(34 \%)$ & $1,6(1,2-2,1)$ & $35,7 \%(13,3 \%-52,4 \%)$ & $<0,05$ \\
\hline $\begin{array}{l}\text { Paridad: } \\
0\end{array}$ & $\begin{array}{c}n(\%) \\
61(48,4)\end{array}$ & $\begin{array}{c}n(\%) \\
250(66)\end{array}$ & 1 & 0 & - \\
\hline 1 & $37(29,4)$ & $97(25,6)$ & $1,4(1-2,0)$ & $29 \%$ (-1\%-50,2\%) & $>0,05$ \\
\hline 2 & $20(15,9)$ & $26(6,9)$ & $2,2(1,5-3,3)$ & $54,9 \%(32,7 \%-69,7 \%)$ & $<0,05$ \\
\hline$\geq 3$ & $8(6,4)$ & $6(1,6)$ & $2,9(1,8-4,8)$ & $65,7 \%$ (43\%-79,3\%) & $<0,05$ \\
\hline $\begin{array}{l}\text { Lesiones de } \\
\text { cuello uterino: }\end{array}$ & n (\%) & n (\%) & & & \\
\hline Ninguno & $119(94,4)$ & $354(93,4)$ & 1 & 0 & \\
\hline Bajo grado & $5(4)$ & $17(4,5)$ & $0,9(0,4-2)$ & $9,7 \%$ (-98,3\%-58,8\%) & \\
\hline Alto grado & $1(0,8)$ & $2(0,5)$ & $1,3(0,3-6,6)$ & $24,5 \%(-276,8 \%-84,9 \%)$ & \\
\hline Otras & $1(0,8)$ & $6(1,6)$ & $0,6(0,1-3,5)$ & $43,2 \%(-250,9 \%-90,8 \%)$ & $>0,05$ \\
\hline
\end{tabular}

Tabla III

EMBARAZOS, PARTOS Y LESIONES DE CUELLO UTERINO EN ADOLESCENCIA TEMPRANA VS TARDÍA

\begin{tabular}{|c|c|c|c|c|c|}
\hline Resultado & $\begin{array}{c}\text { Sexarquia en } \\
\text { adolescencia } \\
\text { temprana } \\
\text { n: } 126\end{array}$ & $\begin{array}{c}\text { Sexarquia en } \\
\text { adolescencia } \\
\text { tardía } \\
\text { n: } 340\end{array}$ & $\mathrm{RR}$ & RAexp \% & $\begin{array}{c}\text { Valor } \\
p\end{array}$ \\
\hline Embarazo n (\%) & $65(51,6 \%)$ & \multirow{2}{*}{$\begin{array}{c}99(29,1 \%) \\
n(\%)\end{array}$} & $2(1,5-2,6)$ & $49 \%(31,7 \%-62 \%)$ & $<0,05$ \\
\hline Paridad: & $\mathrm{n}(\%)$ & & & & \\
\hline 0 & $61(48,4)$ & \multirow{4}{*}{$\begin{array}{c}241(70,9) \\
85(25) \\
13(3,8) \\
1(0,3)\end{array}$} & 1 & 0 & - \\
\hline 1 & $37(29,4)$ & & $1,5(1,1-2,1)$ & $33,4 \%(5,5 \%-53,1 \%)$ & $<0,05$ \\
\hline 2 & $20(15,9)$ & & $3(2,1-4,3)$ & $66,7 \%(52,5 \%-76,6 \%)$ & $<0,05$ \\
\hline$\geq 3$ & $8(6,4)$ & & $4,4(3,2-6,1)$ & $77,3 \%(68,6 \%-83,5 \%)$ & $<0,05$ \\
\hline \multicolumn{6}{|l|}{ Lesiones } \\
\hline $\begin{array}{l}\text { de cuello } \\
\text { uterino: }\end{array}$ & n (\%) & \multirow{2}{*}{$\begin{array}{c}n(\%) \\
314(92,4)\end{array}$} & & & \\
\hline Ninguno & $119(94,4)$ & & 1 & & \\
\hline Bajo grado & $5(4)$ & \multirow{3}{*}{$\begin{array}{c}19(5,6) \\
3(0,9) \\
4(1,2)\end{array}$} & $0,8(0,3-1,7)$ & $24,2 \%(-67,8 \%-65,8 \%)$ & \\
\hline Alto grado & $1(0,8)$ & & $0,9(0,2-5)$ & $9 \%(-400,1 \%-83,5 \%)$ & \\
\hline Otras & $1(0,8)$ & & $0,7(0,1-4,2)$ & $27,2 \%(-323 \%-87,5 \%)$ & $>0,05$ \\
\hline
\end{tabular}




\section{DISCUSIÓN}

La sexualidad y la adolescencia son dos conceptos, que juntos, en nuestra sociedad y ámbito médico ocasionan inquietud por los riesgos que se tienen: embarazo no planificado, enfermedades de transmisión sexual, además de las críticas morales. La adolescencia es un período importante en el proceso de construcción de modelos socio-culturales. Es la etapa más conflictiva en la relación entre padres e hijos. Los adolescentes deben tomar decisiones respecto a la expresión sexual, que va desde la abstinencia total, hasta la máxima promiscuidad (17). Esta fase del desarrollo del joven depende en gran medida de las normas que rigen su sociedad (18). El 19,6\% de los colombianos son adolescentes. Esto exige una adecuada preparación médica para atender los problemas de salud de esta población. En Colombia, la mortalidad por causas externas es elevada para este grupo, pero estas causas de muerte son las principales en adolescentes a nivel mundial. El sentido de invulnerabilidad y las conductas exploratorias favorecen el consumo de sustancias tóxicas (alcohol, 65\%, y tabaco, 35,8\%), la precocidad sexual (embarazo, $21 \%$ ) y la falta de autocontrol (19).

Este trabajo pretendió evaluar la asociación entre inicio temprano de la AS en adolescentes, número de hijos, planificación familiar y presencia de lesiones en cuello de útero de bajo y alto grado. En este grupo de adolescentes y jóvenes, encontramos que el inició de la AS ocurre con un promedio de 16 años ( $\pm 1,6$ años), siendo las edades más tempranas los 11 años (n: 1; 0,1\%), 12 años (n: 8; $1 \%$ ), 13 años (n: 45; 5,3\%), 14 años (n: 72; 8,5\%), 15 años (n: $175 ; 20,7 \%$ ) y 16 años (n: $204 ; 24,1 \%$ ). El $15 \%$ de los adolescentes y jóvenes que acudieron a esta IPS a toma de citología vaginal había iniciado su actividad sexual en la adolescencia temprana y el $44,8 \%$ en la adolescencia media y el 40,2\% en la adolescencia tardía. Para América Latina y El Caribe (ALC), aproximadamente el $50 \%$ de los adolescentes < 17 años son sexualmente activos y un $53-71 \%$ de las mujeres tuvieron relaciones sexuales antes de los 20 años (20). La edad del primer coito es de aproximadamente 15-16 años para las jóvenes de muchos países de ALC, mientras en algunos países del Caribe, inician la vida sexual a una edad tan temprana como los 10-12 años (21).

García y cols (21), hallaron que en adolescentes y jóvenes, el $61 \%$ de los encuestados tenía vida sexual activa; el inicio de las relaciones ocurrió en un $44 \%$ en las edades comprendidas entre los 1519 años, y en el 55\%, antes de los 20 años. Para estos autores, los adolescentes tempranos habían iniciado su AS en un 11\%, mientras los adolescentes medios y tardíos en un $44 \%$ y los jóvenes en un $6 \%$.

Estudios de fecundidad en México, hallaron que para las adolescentes, la mediana de la edad de su primera relación sexual en 1987 fue 17,6 años y para 2009 se desplazó apenas unos meses, para quedar en 17,8 años. Entre las adolescentes, el $50 \%$ ya había experimentado un primer coito a los 15,1 años en 1987, lo que en 2009 ocurría a los 15,4 años $(22,23)$.

El $34,7 \%$ de las adolescentes y jóvenes tenían al menos un hijo, $7 \%$ dos hijos y $1,8 \% \geq 3$ hijos. El $51,6 \%, 34 \%$ y $29,1 \%$ de las evaluadas que habían iniciado la AS entre los 11-14 años, 15-16 años y 17-19 años respectivamente, habían tenido un hijo $(p<0,05)$. Las adolescentes $<15$ años que inician su AS tienen un mayor riesgo de tener hijos cuando se comparan con las que inician la AS entre los 15-16 años (RR: 1,6; IC 95\%:1,2-2,1) y las que la inician entre los 17-19 años (RR: 2; IC 95\%: 1,5$2,6)$. Pero, un hecho más preocupante, es que las que inician la AS en la adolescencia temprana tiene mayor riesgo de multiparidad ( $\geq 3$ hijos) durante la adolescencia y la juventud (10-25 años de edad). En nuestro trabajo 3/192 (1,6\%) adolescentes al momento de la consulta y 12/653 (1,8\%) de las jóvenes entre los 20-25 años habían tenido 3 o más hijos. Asumiendo que muchos de estos embarazos son no planificados y no deseados, si se retrasara el inicio de la actividad sexual de los adolescentes a una edad superior a los 14 años, evitaríamos en un $35,7-49 \%$ los embarazos en adolescentes y jóvenes y la multiparidad en un 65,6-77,3\%.

Indudablemente, cuando se considera a la fecundidad en la adolescencia como un fenómeno a combatir, el área de oportunidad sobre la cual es urgente trabajar es precisamente la fecundidad abiertamente declarada como no planeada o no deseada. En México, estimaciones del Consejo Nacional de Población (CONAPO) con base en la Encuesta Nacional de la Dinámica Demográfica (ENADID) 2006 y 2009 (22), informan que para el grupo de edad de 15-19 años, el 59,4\% de los embarazos fue planificado, el $27,8 \%$ no planificado y el $12,8 \%$ no deseado; mientras que para el grupo de $20-24$ años, el $67,3 \%$ de los embarazos fue planificado, el $23,2 \%$ no planificado y el $9,5 \%$ no deseado. Las estimaciones del CONAPO revelan que en 2009, nueve de cada diez mujeres entre 15-19 años de edad no tienen hijos nacidos vivos, mientras que casi nueve de cada cien tienen uno. Entre las adultas jóvenes estas proporciones sufren cambios radicales: en el mismo año, una de cada cuatro tiene un hijo nacido vivo, una de cada seis tienen dos y 
casi seis de cada cien tienen tres hijos o más; sólo tres de cada diez mujeres llegan a los 25 años de edad sin hijos.

En Colombia, la situación no es mejor. En el grupo de mujeres entre los 15-19 años, el 21\% ya son madres o están embarazadas de su primer hijo, lo que significa que una de cada cinco adolescentes ya ha iniciado su ciclo de reproducción. Esta situación es más grave en víctimas del desplazamiento, $63 \%$ de las cuales han estado o están embarazadas de su primer hijo antes de cumplir los 19 años. Pero un hecho alarmante, es que entre las primeras causas de muerte en adolescentes entre 15-19 años están las complicaciones del embarazo, parto y puerperio, mientras que entre las causas de enfermedad para este grupo de mujeres, los primeros lugares son ocupados por entidades relacionadas con el comportamiento sexual (19). La tasa de fecundidad en adolescentes mientras ha disminuido en la mayoría de los países de América Latina, en Colombia aumentó de 70 a 90 por mil entre 1986 y 2005, y la proporción de adolescentes alguna vez embarazada pasó de $19 \%$ a $21 \%$ entre 2000 y 2005, una relación inversa con el nivel de educación y el índice de riqueza (19).

Otro aspecto hallado muy importante, es que entre todas las adolescentes y jóvenes del estudio, el $49 \%$ no utilizaban ningún método de planificación familiar. Mientras, el 34\% planificaban con hormonas, sólo el $5 \%$ usaban métodos de barrera como método de planificación y protección contra enfermedades de transmisión sexual.

Nuestros hallazgos contrastan con los de García y cols (21), donde el preservativo no se usó nunca en el $30 \%$ de las encuestadas y ocasionalmente en un $49 \%$, mientras el $21,3 \%$ siempre lo usaban, cifra más altas que las de este trabajo. Estos hallazgos contrastan con los informados por otros autores (20), donde solamente el $30 \%$ de los jóvenes del Caribe se preocupan del embarazo. El $26 \%$ utilizan anticonceptivos de forma constante. Las encuestas en El Caribe sugieren que el $40 \%$ de las adolescentes no tienen acceso a anticonceptivos durante su primera relación sexual. El $35-52 \%$ de los embarazos en la Región no fueron planificados, el 38\% de las mujeres quedan embarazadas antes de los 20 años y para América Latina entre el 15-25\% de todos los recién nacidos eran hijos de adolescentes (20). Un estudio realizado en México en 2009 (22), halló que las adolescentes que iniciaron su primera relación sexual antes de los 15 años el $75 \%$ no utilizó ningún método de planificación, el 23,4\% condón, el $0,8 \%$ método hormonal y el $0,7 \%$ otros, mientras que las que iniciaban su primera relación entre los 15-19 años el 58,9\% no utilizó ningún mé- todo de planificación, 36,4\% condón, 3\% método hormonal y $1,7 \%$ otro.

Para Colombia, la edad promedio del inicio de la actividad sexual, es de 18,3 años. Sin embargo, $11 \%$ de las mujeres inician su primera relación antes de los 15 años y existen diferencias significativas de acuerdo con el nivel de educación: 16 años, en mujeres sin educación, y 20 años en nivel superior. Se estima que $21 \%$ de la población de 15-19 años es activa sexualmente, pero el $63 \%$ no usa ningún método de anticoncepción. A pesar de que el conocimiento de la existencia de métodos anticonceptivos es universal en mujeres entre los 13-49 años, sólo $81 \%$ ha usado un método alguna vez en su vida y $10 \%$ lo usan en su primera relación sexual. Los métodos más utilizados por adolescentes son la píldora, el condón, el coito interrumpido y el ritmo. Pero, cerca de $41 \%$ de las usuarias del método del ritmo, desconocen los días de riesgo y $42 \%$ de las mujeres que usan anticonceptivos los suspenden luego del primer año (19).

Por otro lado, el 93,1\% de las evaluadas no presentaron ninguna lesión de cuello uterino, mientras, el 4,9\% tenían lesiones de bajo grado y el 0,7\% lesiones de alto grado. Sin embargo, no se halló diferencia estadísticamente significativa entre las que habían iniciado su AS en la adolescencia temprana, media y tardía. En un estudio con 9.657 mujeres en los Estados Unidos de Norte América (USA) la prevalencia del papiloma virus (VPH) de alto riesgo en adolescentes de 14-19 años fue de 35\% (IC 95\%: 32-38) (24). Aunque la neoplasia intracervical III es muy baja en mujeres menores de 20 años, la prevalencia de la positividad para el VPH de alto riesgo es menor en las mujeres con citologías normales que en aquellos con resultados anormales. Sin embargo, Peto y cols (25), hallaron que entre mujeres de 15-19 años, el 17\% con citologías normales y el $74 \%$ con resultados anormales tuvieron pruebas positivas para el VPH de alto riesgo.

Otro estudio realizado en el Reino Unido (26), con una cohorte de 1.075 mujeres de 15-19 años con resultados de citología normal y negativos para VPH de alto riesgo, fueron seguidas durante 29 meses mediante frotis vaginal y pruebas de VPH, encontrando que el $26 \%$ de las mujeres se convirtió en positivo para 6 tipos de VPH de alto riesgo, siendo la mediana de la duración de los primeros casos positivos de VPH de 13,7 meses para cualquier tipo de VPH, 10,3 meses para el VPH 16, y 7,8 meses para el VPH 18. El acumulado de 3 años de riesgo para cualquier anormalidad citológica fue del 28\% (IC 95\%: 25-32). En esta cohorte, 28 mujeres $(2,6 \%)$ desarrollaron alteraciones, neoplasia intracervical $2(1,3 \%)$ o neoplasia intracervical $3(1,3 \%)$ 
durante una mediana de 36 meses de seguimiento.

Más allá de los embarazos, están los abortos que no fueron materia de este estudio, lo mismo que las enfermedades de transmisión sexual. La mortalidad materna sigue siendo una de las causas principales de muerte entre las adolescentes. En Chile y Argentina, donde el aborto está prohibido, más de la tercera parte de las muertes de madres adolescentes son consecuencia directa de prácticas de aborto. Entre $21-30 \%$ de los embarazos en México, Colombia, Brasil, República Dominicana, Chile y Perú terminan en aborto (20). Por otro lado, cada año, el 15\% de los adolescentes entre 15-19 años de edad adquieren enfermedades de transmisión sexual, principal causa de infecciones del tracto reproductivo. En Haití, se estima que el 4,9\% de los jóvenes varones entre 15-24 años de edad están viviendo con VIH (20).

Las limitaciones de este estudio son que se trabajó con datos retrospectivos, que fueron recogidos para otros fines diferentes a este estudio, lo cual ha podido ocasionar fallas en los registros clínicos. Esto se pudo traducir en sesgos de información, sin embargo, conocemos de la rigurosidad de la entidad Unicáncer Capítulo Tuluá en la recolección de esta información. Por otro lado, carecemos de otra información importante como escolaridad, estado civil, ocupación, seguridad social en salud, vacunación contra VPH, entre otras variables, que hubieran sido importantes de analizar como asociadas a las variables analizadas. Las perspectivas futuras de investigación es la realización de un trabajo prospectivo que permita determinar los factores que están asociados al inicio precoz de la actividad sexual en los adolescentes, así como sus consecuencias en cuanto a embarazos y enfermedades de transmisión sexual, entre otros aspectos.

\section{CONCLUSIONES}

De acuerdo a nuestros resultados se comprueba el inicio precoz de la actividad sexual en adolescentes y jóvenes que consultan en una IPS colombiana para tamización de cáncer de cuello uterino, como también el bajo uso de métodos anticonceptivos efectivos. Esto hace que el $34,7 \%$ de las adolescentes tengan al menos 1 hijo y el 6,9\% tenga alguna lesión cervical. Programas educativos conducentes al retraso del inicio de la actividad sexual, el uso de métodos anticonceptivos efectivos y de barrera, permitirán reducir las cifras encontradas en este estudio.
AGRADECIMIENTOS: Los integrantes del grupo de investigación agradecen a los ejecutivos de Unicáncer Capítulo Tuluá, Colombia y a los dirigentes de la Unidad Central del Valle (UCEVA), Colombia, por sus desinteresados esfuerzos en brindar la colaboración necesaria para la realización del presente trabajo.

\section{REFERENCIAS}

1. Issler JR. Embarazo en la adolescencia. Revista de posgrado de la VI cátedra de Medicina 2001;107:1123.

2. Serra FG, Spaciuk SM, Morales E, Arce GM, Avalos ME, Gómez VY. Riesgo reproductivo del adolescente en Corrientes. Revista de posgrado de la VI cátedra de Medicina 2002;115:24-5.

3. Balestena JM, Balestena SG. Impacto de la menarquía en los resultados maternos perinatales en la adolescencia. RCOG [en línea] 2005 [2 de enero de 2012]; 31(1).URL disponible en: http://www.bvs.sld. cu/revistas/gin/vol31_1_05/gin02105. htm

4. Laffita A, Ariosa JM, Cutié JR. Adolescencia e interupciones del embarazo. RCOG [en línea] 2004 [2 de enero de 2012]; 30(1).URL disponible en: http://www. bvs.sld.cu/revistas/gin/vol30_1_04/gin04104. htm

5. Valdés S, Essien J, Bardales J, Saavedra D, Bardales E. Embarazo en la adolescencia. Incidencia, riesgos y complicaciones. RCOG. [en línea] 2002 [2 de enero de 2012 de noviembre de 2005]; 28(2). URL disponible en: http://www.bvs.sld.cu/revistas/gin/vol28_2_02/ gin04202. htm

6. Fernández LS, Carro E, Osesa D, Pérez J. Caracterización del recién nacido en una muestra de gestantes adolescentes. RCOG [en línea] 2004 [2 de enero de 2012]; 30(2). URL disponible en: http://www.bvs.sld. cu/revistas/gin/vol30_2_04/gin03204. htm.

7. Romero MI, Maddaleno M, Silber TJ, Munist M. Salud reproductiva. En: Silber TJ, Munist MM, Maddaleno M, Suarez Ojeda. EN: Manual de medicina de la adolescencia. EEUU: Publicación de la OPS 1992:473-518.

8. Cutié JR, Laffita A, Toledo M. Primera relación sexual en adolescentes cubanos. Rev Chil Obstet Ginecol 2005;70:83-6.

9. Marchetti R, Rodríguez G, Rodríguez A. Los adolescentes y el SIDA: La importancia de la educación para la prevención. SIDA-FTS/1996;2:61-9.

10. Juárez $F$, Gayet $C$. Salud sexual y reproductiva de los adolescentes en México: Un nuevo marco de análisis para la evaluación y diseño de políticas. Papeles de la Población 2005;45:177-219. Visitado Enero 20 de 2011. Disponible en: http://www.redalyc.org/redalyc/ pdf/112/11204508.pdf

11. Langer Ana. El embarazo no deseado: impacto sobre la salud y la sociedad en América Latina y el Caribe. Rev Panam Salud Publica 2002;11:192-205.

12. Tavara L. Contribución de las adolescentes a la muerte materna en el Perú. Ginecol Obstet Perú 2004;50:111-22. 
13. Lezcano SA, Vallejos MS, Sodero H. Caracterización del recién nacido en madres adolescentes. Revista de Posgrado de la VI Cátedra de Medicina 2005;149:6-8.

14. Donoso E. Embarazo adolescente: un problema país. Rev Chil Obstet Ginecol 2008;73:291-2.

15. Donoso E. Aumento de la natalidad en Chile: ¿cambio demográfico deseable? Rev Chil Obstet Ginecol 2009;74:133-4.

16. Solomon D, Davey D, Kurman R, et al. The 2001 Bethesda System: terminology for reporting results of cervical cytology. JAMA 2002;287:2114-9.

17. Pick S, Givaudan M, Saldívar-Garduño A. La importancia de los factores psicosociales en la educación sexual de los adolescentes. Perinatol Reprod Hum 1996;10:143-50.

18. García I, Ávila E, Lorenzo A, Lara M. Conocimientos de los adolescentes sobre aspectos de la sexualidad. Rev Cubana Pediatr 2002;74(4).

19. Alba LH. Salud de la adolescencia en Colombia: bases para una medicina de prevención. Univ Méd Bogotá (Colombia) 2010;51:29-42.

20. Schutt-Aine J, Maddaleno M. (2003). Salud sexual y desarrollo de adolescentes y jóvenes en las Américas: Implicaciones en programas y políticas. (sitio en Internet). OPS, Washington, DC. Visitado Enero 20 de 2011. Disponible: www.paho.org/Spanish/HPP/HPF/ ADOL/SSRA.pdf.

21. Garcia R, Cortés A, Vila L, Hernández M, Mesquia A. Comportamiento sexual y uso del preservativo en adolescentes y jóvenes de un área de salud. Rev Cubana Med Gen Integr 2006;22(1). Visitado Enero 20 de 2011. Disponible en: bvs.sld.cu/revistas/mgi/ vol22_1_06/mgi03106.pdf

22. Consejo Nacional de Población (CONAPO). Estimaciones del CONAPO con base en Encuesta Nacional de Fecundidad y Salud 1987, Encuesta Nacional de Salud Reproductiva 2003 y Encuesta Nacional de la Dinámica Demográfica 2006 y 2009. Visitado Enero 20 de 2011. Disponible en: www.conapo.gob.mx/publicaciones/CuaTec/pdf/tendenciasfecun.pdf.

23. Colin Y, Villagómez P. Evolución de la maternidad adolescente en México, 1974-2009. La situación demográfica en México 2010. Pag 37-53. Visitado Enero 20 de 2011. Disponible en: http://www.conapo.gob. $\mathrm{mx} /$ publicaciones/sdm/sdm2010/03.pdf

24. Datta SD, Koutsky LA, Ratelle S, Unger ER, Shlay J, McClain $\mathrm{T}$, et al. Human papillomavirus infection and cervical cytology in women screened for cervical cancer in the United States, 2003-2005. Ann Intern Med 2008;148:493-500.

25. Peto J, Gilham C, Deacon J, Taylor C, Evans C, Binns W, et al. Cervical HPV infection and neoplasia in a large population-based prospective study: the Manchester cohort. Br J Cancer 2004;91:942-53.

26. Woodman CB, Collins S, Winter H, Bailey A, Ellis J, Prior $\mathrm{P}$, et al. Natural history of cervical human papillomavirus infection in young women: a longitudinal cohort study. Lancet 2001;357:1831-6. 